\title{
Treatment outcomes of extended-field radiation therapy for thoracic superficial esophageal cancer
}

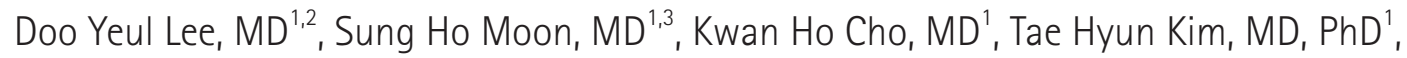 \\ Moon Soo Kim, MD³, Jong Yeul Lee, $\mathrm{MD}^{3}$, Yang-Gun Suh, MD, PhD ${ }^{1,2,3}$ \\ 'Proton Therapy Center, Research Institute and Hospital, National Cancer Center, Goyang; \\ ${ }^{2}$ Department of Cancer Biomedical Science, National Cancer Center Graduate School of Cancer Science and Policy, Goyang; \\ ${ }^{3}$ Center for Lung Cancer, Research Institute and Hospital, National Cancer Center, Goyang, Korea
}

Purpose: To evaluate the efficacy and safety of extended-field radiation therapy for patients with thoracic superficial esophageal cancer (SEC).

Materials and Methods: From May 2007 to October 2016, a total of 24 patients with thoracic SEC (T1a and T1b) who underwent definitive radiotherapy and were analyzed retrospectively. The median total radiotherapy dose was 64 Gy (range, 54 to $66 \mathrm{~Gy}$ ) in conventional fractionation. All 24 patients received radiotherapy to whole thoracic esophagus and 23 patients received elective nodal irradiation. The supraclavicular lymph nodes, the celiac lymph nodes, and both of those nodal areas were included in 11,3 , and 9 patients, respectively.

Results: The median follow-up duration was 28.7 months (range 7.9 to 108.0 months). The 3-year overall survival, local control, and progression-free survival rates were $95.2 \%, 89.7 \%$, and $78.7 \%$, respectively. There were 5 patients $(20.8 \%)$ with progression of disease, 2 local failures (8.3\%) and $3(12.5 \%)$ regional failures. Three patients also experienced distant metastasis and had died of disease progression. There were no treatment-related toxicities of grade 3 or higher.

Conclusion: Definitive extended-field radiotherapy for thoracic SEC showed durable disease control rates in medically inoperable and endoscopically unfit patients. Even extended-field radiotherapy with elective nodal irradiation was safe without grade 3 or 4 toxicities.

Keywords: Esophageal neoplasms, Radiotherapy, Endoscopic mucosal resection

\section{Introduction}

Esophageal cancer is the 8th most common cancer worldwide and the 6th most common cause of cancer-related death worldwide [1]. The detection rate of superficial esophageal cancer (SEC), which is defined as a tumor limited to the mucosa or submucosa, has been increasing by the widespread endoscopic screening and the development of new endoscopic techniques [2-4]. Recently, endoscopic resection is recognized as a possible curative option for SEC confined within the mucosal epithelium or the lamina propria mucosa (T1a) $[5,6]$, however, submucosal tumors (T1b) or large mucosal tumors are considered to be not suitable for endoscopic resection. In addition, pT1 esophageal cancer is frequently accompanied with a lymph node metastasis with 5-year metastasis rate of 5.7\% (0.4\%-8.7\% for T1a and 25.7\% for T1b) [7]. As a

Received 14 September 2017, Revised 19 September 2017, Accepted 20 September 2017.

Correspondence: Yang-Gun Suh, MD, PhD, Proton Therapy Center, Research Institute and Hospital, National Cancer Center, 323 Ilsan-ro, Ilsandong-gu, Goyang 10408, Korea. Tel: +82-31-920-1722, Fax: +82-31-920-0149, E-mail: suhmd@ncc.re.kr

(c) This is an Open Access article distributed under the terms of the Creative Commons Attribution Non-Commercial License (http://creativecommons.org/ licenses/by-nc/4.0/) which permits unrestricted non-commercial use, distribution, and reproduction in any medium, provided the original work is properly cited.

www.e-roj.org 
result, esophagectomy with lymph node dissection has been considered as the standard treatment for SEC, however, esophagectomy is highly invasive and accompanied with increased morbidity and mortality rates $[8,9]$.

Instead of surgery, concurrent chemoradiotherapy (CCRT) or radiotherapy alone can be an alternative option. Several studies have reported favorable outcomes of CCRT or radiotherapy alone for SEC patients with the local control (LC) rates of $70 \%$ to $80 \%$ [10-13]. However, the use of concurrent chemotherapy, radiation field, and radiation dose remain unclear.

In the present study, we investigated the treatment outcomes of radiotherapy alone with extended-field radiation for patients with SEC.

\section{Materials and Methods}

\section{Patients}

Between May 2007 and October 2016, a total of 24 patients with SEC who underwent radiotherapy with or without endoscopic resection at our institution were included in this study (Table 1). Pretreatment evaluation included a medical history and physical examination. Laboratory studies included complete blood cell count and blood chemistries. For stage work-up, esophagogastroduodenoscopy (EGD) with Lugol staining, chest computerized tomography (CT) and endoscopic ultrasonography (EUS) were performed. To evaluate the distant metastasis, patients were evaluated by $18 \mathrm{~F}$-fluorodeoxyglucose positron emission tomography (FDG-PET), bone scans, and abdomen CT. Clinical stage was based on the 7th edition of the American Joint Committee on Cancer (AJCC) TNM classification.

\section{Radiotherapy}

Radiotherapy was delivered using megavoltage photon beam or proton beam. A conventional fractionation schedule with a fraction size of 2 Gy was used in all patients. Threedimensional conformal radiotherapy (3D-CRT), intensitymodulated radiotherapy (IMRT), and proton beam therapy (PBT) was used in 17, 4, and 3 patients, respectively. The gross tumor volume (GTV) was defined by the tumor visualized on CT, FDGPET or the area where were marked within $1 \mathrm{~cm}$ proximal and distal to the primary tumor site using endoscopic clips marked by an endoscopy specialist before taking a planning CT. For all 24 patients, the initial clinical target volume (CTV) included the whole thoracic esophagus (from the upper esophageal sphincter to the esophagogastric junction). Elective nodal areas were included in the initial CTV except in 1 patient. The
Table 1. Patients' characteristics $(n=24)$

\begin{tabular}{|c|c|}
\hline Characteristic & Value \\
\hline Age (yr) & 71.5 (39-81) \\
\hline$\geq 70$ & $13(54.2)$ \\
\hline$<70$ & $11(45.8)$ \\
\hline \multicolumn{2}{|l|}{ Gender } \\
\hline Male & $21(87.5)$ \\
\hline Female & $3(12.5)$ \\
\hline \multicolumn{2}{|l|}{ ECOG performance status } \\
\hline $0-1$ & $22(91.7)$ \\
\hline 2 & 2 (8.3) \\
\hline \multicolumn{2}{|l|}{ Tumor location } \\
\hline Middle thoracic & $18(75.0)$ \\
\hline Lower thoracic & $6(25.0)$ \\
\hline \multicolumn{2}{|l|}{ T stage } \\
\hline T1a & 4 (16.7) \\
\hline $\mathrm{T} 1 \mathrm{~b}$ & $20(83.3)$ \\
\hline Tumor size (cm) & $2.5(0.6-6.2)$ \\
\hline$\geq 2.5$ & $13(54.2)$ \\
\hline$<2.5$ & $11(45.8)$ \\
\hline \multicolumn{2}{|l|}{ ESD } \\
\hline No & $10(41.7)$ \\
\hline Yes & $14(58.3)$ \\
\hline
\end{tabular}

Values are presented as median (range) or number (\%). ECOG, Eastern Cooperative Oncology Group; ESD, endoscopic submucosal dissection.

supraclavicular lymph nodes (SCN), the celiac lymph nodes, and both of those nodal areas were included in 20, 12, and 9 patients, respectively. In patients with middle thoracic SEC, SCN and celiac lymph nodes were irradiated in 88.9\% and $61.2 \%$ of cases, respectively. In the patients with lower thoracic SEC, SCN and celiac lymph nodes were included in the radiation field for $66.7 \%$ and $83.3 \%$ of cases, respectively. A shrinking field technique was used in 23 patients except the patient treated with a total radiation dose of $54 \mathrm{~Gy}$. The boost CTV encompassed the primary tumor with a margin of at least $1 \mathrm{~cm}$ for all directions. The planning treatment volume (PTV) was created by adding $0.5-1 \mathrm{~cm}$ of margin to the CTV. The initial PTV was treated with 40-54 Gy (median dose, 44 Gy). To reduce lung irradiation, anterior-posterior parallel opposite fields were used in the initial phase. To restrict the spinal cord dose, anterior and posterior oblique fields were used for the boost phase. For the patients underwent endoscopic submucosal dissection (ESD), total radiation doses of 54-66 Gy were administered, whereas total radiation doses of 60-66 Gy were administered for the patients received radiotherapy alone. As a result, the total radiation dose ranged between 54 and 66 Gy (median dose, 64 Gy). Variables related to radiotherapy are 
Table 2. Radiation therapy

\begin{tabular}{lc}
\multicolumn{1}{c}{ Characteristic } & Value \\
\hline Total radiation dose (Gy) & $64(54-66)$ \\
Radiation dose for initial PTV (Gy) & $44(40-54)$ \\
SCN irradiation & \\
No & $4(16.7)$ \\
Yes & $20(83.3)$ \\
Celiac LN irradiation & $12(50.0)$ \\
No & $12(50.0)$ \\
Yes & $1(4.2)$ \\
RT field & $11(45.8)$ \\
Whole esophagus & $3(12.5)$ \\
Whole esophagus + SCN & $9(37.5)$ \\
Whole esophagus + celiac & \\
Whole esophagus + SCN + celiac & $17(70.8)$ \\
RT modality & $4(16.7)$ \\
3D-CRT & $3(12.5)$ \\
IMRT & \\
Proton beam therapy &
\end{tabular}

Values are presented as median (range) or number (\%).

PTV, planning target volume; SCN, supraclavicular lymph nodes; LN, lymph nodes; RT, radiotherapy; 3D-CRT, 3-dimensional conformal radiotherapy; IMRT, intensity-modulated radiotherapy.

summarized in Table 2.

\section{Follow-up and statistical analysis}

During radiotherapy, patients were examined weekly to check treatment-related toxicities. Patients were followed up with EGD or chest CT every 3 months in the first 2 years, and every 6 months thereafter. Treatment related toxicities were graded according to the Common Terminology Criteria for Adverse Events (CTCAE) version 4.0. Treatment responses were evaluated according to the Revised Response Evaluation Criteria in Solid Tumors (RECIST) guideline version 1.1. Survival duration was calculated from the date of initiation of radiotherapy to the last follow-up or the considered events (local failure, regional failure, distant failure, or death). Survival rates were calculated using the Kaplan-Meier method. The log-rank test was used to assess the statistical differences in Kaplan-Meier estimates. Differences in clinical features between patients treated with ESD followed by radiotherapy and radiotherapy alone were analyzed using the Pearson chisquare or independent samples t-tests. Statistical analyses were conducted using SPSS ver. 18.0 (SPSS Inc., Chicago, IL, USA).

\section{Results}

\section{Patient characteristics and clinical profile}

Pretreatment patients and tumor characteristics are listed on Table 1. Median age was 71.5 years (range, 39 to 81 years), with 21 males (87.5\%) and 3 females (12.5\%). The tumor location of 18 patients (75\%) was mid-thoracic esophagus and the other 6 patients $(25 \%)$ had tumor in lower thoracic esophagus. Of the 24 patients, 10 patients (41.7\%) received radiotherapy for the first-line treatment, because they were not candidate for endoscopic resection due to submucosal invasion seen in EUS. Eight patients were medically unfit to undergo esophagectomy due to old age, heart diseases, cerebrovascular disease, and poor lung function, while 2 patients strongly refused to take surgery. The remaining 14 patients (58.3\%), who initially presented with T1a diseases in EUS, underwent radiotherapy after ESD because of their pathologic features including the invasion of tumors into muscularis mucosae (M3 according to the Japanese subclassification $[14,15])$ or submucosa (T1b).

\section{Treatment outcomes}

The median follow-up period was 28.7 months (range, 7.9 to 108.0 months). The 3 -year overall survival (OS), LC, and progression-free survival (PFS) rates were 95.2\%, 89.7\%, and $78.7 \%$, respectively (Fig. $1 \mathrm{~A}-1 \mathrm{C}$ ). Among the 24 patients, 5 patients (20.8\%) experienced disease progression, and 2 patients $(8.3 \%)$ had died at the time of analysis. These 2 patients died from disease progression. Local failure and regional failure developed in 2 patients (8.3\%) and 3 patients (12.5\%), respectively. Among the 3 patients experienced regional recurrence, 2 patients also presented with distant metastasis. Of the 5 patients presenting with disease progression, salvage surgery, radiotherapy, CCRT, and chemotherapy were performed in 1, 1, 1, and 2 patients, respectively. Among them, 2 patients who treated with surgery and CCRT, respectively, remained without evidence of disease. The details of patients who experienced recurrences is summarized in Table 3. The patients treated with radiotherapy following ESD showed better PFS compared with the patients treated with radiotherapy alone (3-year PFS rate: $90.0 \%$ vs. $58.3 \% ; p=0.016)$. This result is shown in Fig. 1D. The proportion of initial clinical T1a disease was higher in the patients treated with ESD followed by radiotherapy than the patients treated with radiotherapy alone (100\% vs. 10\%; $p=$ 0.001). However, the proportion of T1a disease at final stage $(16.7 \%$ vs. $10 \% ; p=0.459)$ and median tumor size $(2.1 \mathrm{~cm}$ vs. $3 \mathrm{~cm} ; p=0.601)$ did not show significant differences between 

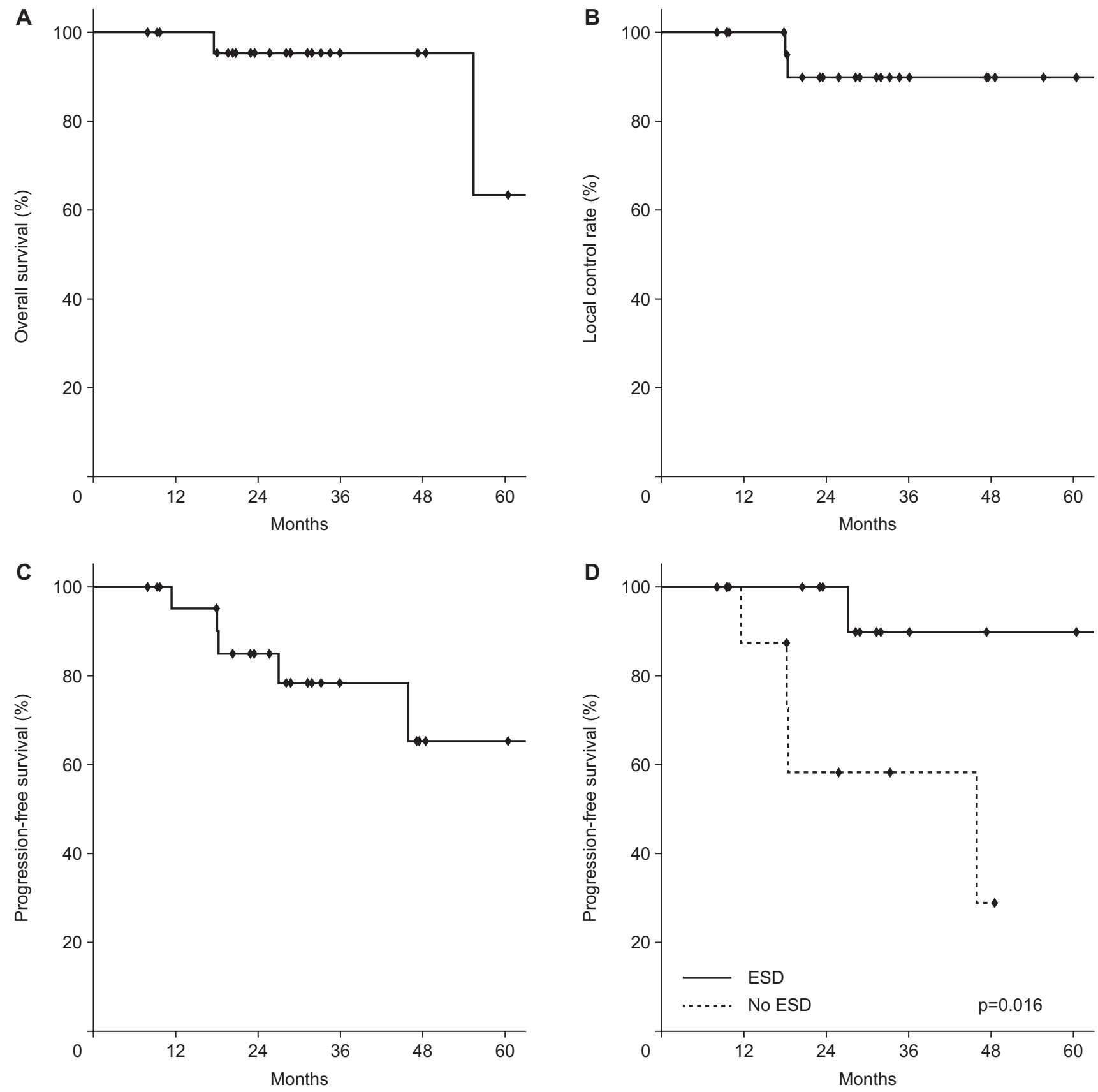

Fig. 1. Kaplan-Meier plot of (A) overall survival, (B) local control, (C) progression-free survival of all patients, and (D) progression-free survival according to the endoscopic submucosal dissection (ESD).

the patients treated with ESD followed by radiotherapy and radiotherapy alone. The 4 patients with T1a disease did not present with recurrence.

\section{Toxicities}

No grade 3 or higher toxicities were observed during the acute and chronic phases. Acute grade 2 esophagitis was developed in 6 patients (25\%) during treatment. Four patients (16.7\%) experienced stenosis of esophagus after treatment, 2 for grade 1 and 2 for grade 2 . These 2 patients with grade 2 esophageal stenosis underwent radiotherapy following ESD and had received intervention with balloon dilatation. After the treatment, grade 1 radiation pneumonitis was observed in 12 patients (50\%) and there was no grade 2 or 


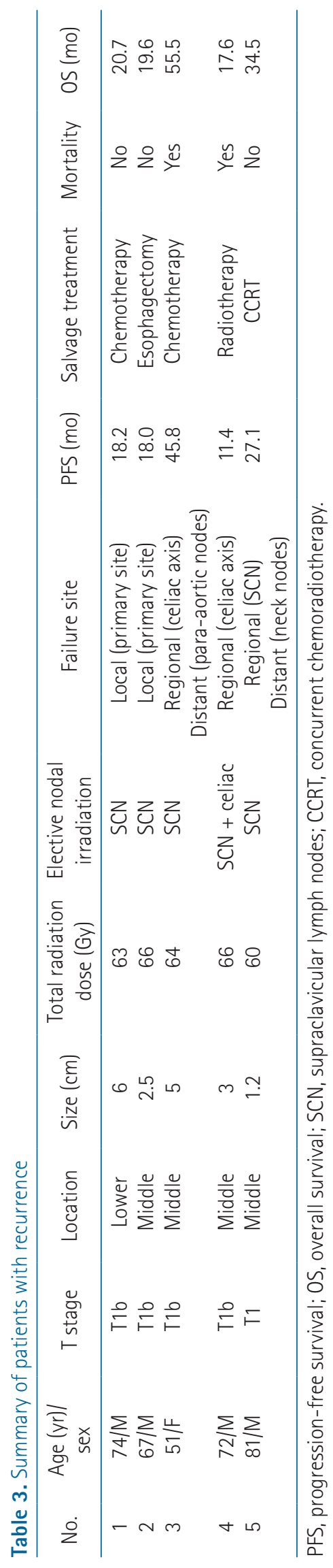

higher radiation pneumonitis. Among the patients, who experienced radiation pneumonitis, 11 patients received the total radiation dose of $60 \mathrm{~Gy}$ or more.

\section{Discussion and Conclusion}

The number of patients with SEC is increasing particularly in Asia due to screening for upper gastrointestinal tract cancers $[16,17]$. Esophagectomy has been considered as the standard treatment for localized esophageal cancer as well as SEC. Recently, to avoid treatment-related morbidity and mortality of esophagectomy, endoscopic approach is increasingly used for SEC. However, due to high rate of lymphatic metastasis in esophageal cancer even in $\mathrm{T} 1$ lesions, the application of endoscopic resection is highly limited to small T1 lesions without evidence of lymphovascular invasion $[18,19]$. Therefore, esophagectomy is still the standard treatment for patients with SEC invading the submucosa. However, definitive radiotherapy with or without concurrent chemotherapy can be considered for unfit patients for surgery, because esophagectomy can result in long-term complications including dysphagia, reflux, and deterioration of quality of life $[20,21]$.

Oh et al. [13] reported the treatment outcomes of hypofractionated definitive radiotherapy of $60 \mathrm{~Gy}$ with a daily dose of 3 Gy per fraction without chemotherapy for patients with esophageal cancer. In their study, 25 patients had SEC among the total of 70 patients, and the 2 -year OS and LC rates were $80.0 \%$ and $81.6 \%$, respectively for patients with SEC. Koide et al reported the treatment outcomes of CCRT for 123 patients with SEC. In their study, the 5-year OS, PFS, and LC rates were 77\%, 47\%, and $63 \%$, respectively [22]. In these studies, radiotherapy was delivered to involved-field around gross tumors without elective nodal irradiation. Esophageal cancer frequently presents as a multicentric disease or submucosal skip spread due to the rich submucosal lymphatics in the esophagus. Therefore, in RTOG 85-01, extended-radiation field encompassing from the supraclavicular region to the gastroesophageal junction was used [23]. However, the results of RTOG 85-01 were not superior than RTOG 94-05, which adopted involved-field radiation field with 5-cm longitudinal margins and a 2-cm radial margin [24]. One prospective randomized clinical trial that compared extended-and involved-field for cervical and upper thoracic esophageal cancer demonstrated there was no significant difference in median survival between involved- 


\section{Doo Yeul Lee, et al}

and extended-field radiation [25]. Generally, extended-field radiation has been considered that potential for treatmentrelated morbidity is higher than involved-field radiation. As a result, recently, majority of institutions adopted involved-field radiation with concurrent chemotherapy for SEC.

The treatment outcomes of patients with thoracic esophageal cancer remains poor due to the high incidence of lymph node metastasis [26]. Therefore, our policy is to include whole esophagus, SCN, and celiac lymph nodes for all cases in the setting of radiotherapy alone for esophageal cancer. However, we did not include SCN or celiac lymph nodes in the radiation field, when the risk of radiation-induced toxicities due to wide radiation fields was expected to be higher than clinical benefits of wide radiation fields. A previous study demonstrated the pattern of lymph node metastasis in patients with thoracic esophageal squamous cell carcinoma [27]. According to this study, the risk for SCN metastasis in middle and lower thoracic esophageal cancer and the risk for celiac lymph node metastasis in upper thoracic esophageal cancer were relatively low less than $5 \%$. As a result, it may be possible to omit irradiation for SCN or celiac lymph nodes according to the site and extent of disease.

In the present study, we used extended-field radiation, which includes whole esophagus and para-esophageal lymph nodes with or without SCN and celiac axis, without chemotherapy. The concurrent use of chemotherapy during radiotherapy can result in increased toxicities. In RTOG 85-01 study, 10\% of patients treated with CCRT experienced acute life-threatening toxicities or treatment-related mortality (TRM), while $2 \%$ of patients treated with radiotherapy alone experienced acute life-threatening toxicities without TRM. In addition, in the previous study, which tested hypofractionated radiotherapy with a total radiation dose of $60 \mathrm{~Gy}$, no patients with clinical T1-2 disease presented with grade 3 or higher toxicities, while $10 \%$ of all patients experienced those [13]. This finding suggests that the patients with more advanced stages can experience more severe radiation-induced toxicities. In our study, extended-field radiotherapy without chemotherapy produced durable outcomes with a 3-year PFS rate of 78.7\% without excessive toxicities. There was no grade 3 adverse event, and acute and chronic radiation pneumonitis and esophagitis were acceptable. Consequently, we suggest that extended-field radiotherapy can be a good treatment option for SEC, especially in medically unfit patients for surgery or chemotherapy. Moreover, the use of advanced radiotherapy techniques such as IMRT or PBT has been continuously increasing. IMRT and PBT provide better radiation dose
Radiation Oncology Journal ROJ

distribution to the target and reduced radiation doses to normal tissues including lungs, heart, and trachea. Therefore, adverse events of extended-field irradiation can be reduced by the use of IMRT and PBT. Consequently, the therapeutic benefit of the extended-field radiotherapy and omission of chemotherapy may be greater than the potential risk of adverse events by extended-field radiotherapy.

In our study, the treatment outcomes were superior in patients who received radiotherapy following ESD than patients who received radiotherapy alone. The patients treated with ESD followed by radiotherapy presented with higher proportion of T1a disease and smaller tumor size, however these differences were not statistically significant. The reason why the patients treated with ESD followed by radiotherapy showed better PFS than the patients treated with radiotherapy alone may be due to the earlier clinical stage and exact T stage in patients who underwent ESD. In addition, grade 2 esophageal stenosis to require endoscopic treatments is occurred in two patients received ESD, while there was no grade 2 or higher esophageal stenosis in patients received radiation without ESD.

Our study has a limitation of non-randomized, retrospective analysis, and small number of patients. However, given that esophagectomy is the standard treatment for SEC, our number of patients is reasonable. In addition, we cannot analyze the prognostic value of $T$ stage, because the number of patients with T1a was small. To investigate the benefits of extendedfield radiation without chemotherapy, further randomized trials comparing the extended-field radiotherapy and the involved-field radiotherapy with concurrent chemotherapy will be needed.

In conclusion, our study showed that the extendedfield radiation therapy without chemotherapy for medically inoperable and endoscopically unfit SEC patients produced reasonable treatment outcomes without significant toxicities of grade 3 or higher. We will continue to investigate, apply, and monitor the extended-field radiotherapy using advanced radiation technology such as IMRT or PBT for patients with SEC.

\section{Conflict of Interest}

No potential conflict of interest relevant to this article was reported. 


\section{References}

1. Ferlay J, Shin HR, Bray F, Forman D, Mathers C, Parkin DM. Estimates of worldwide burden of cancer in 2008: GLOBOCAN 2008. Int J Cancer 2010;127:2893-917.

2. Kumagai Y, Monma K, Kawada K. Magnifying chromoendoscopy of the esophagus: in-vivo pathological diagnosis using an endocytoscopy system. Endoscopy 2004;36:590-4.

3. Yoshida $T$, Inoue $H$, Usui $S$, Satodate $H$, Fukami N, Kudo SE. Narrow-band imaging system with magnifying endoscopy for superficial esophageal lesions. Gastrointest Endosc 2004;59:288-95.

4. Tachimori $Y$, Ozawa $S$, Numasaki $H$, et al. Comprehensive registry of esophageal cancer in Japan, 2009. Esophagus 2016;13:110-137.

5. Oyama T, Tomori A, Hotta K, et al. Endoscopic submucosal dissection of early esophageal cancer. Clin Gastroenterol Hepatol 2005;3(7 Suppl 1):S67-70.

6. Lee HJ, Lee H, Park JC, Shin SK, Lee SK, Lee YC. Treatment strategy after endoscopic resection of superficial esophageal squamous cell carcinoma: a single institution experience. Gut Liver 2015;9:714-9.

7. Yamashina $T$, Ishihara $R$, Nagai $K_{1}$ et al. Long-term outcome and metastatic risk after endoscopic resection of superficial esophageal squamous cell carcinoma. Am J Gastroenterol 2013;108:544-51.

8. Griffin SM, Shaw IH, Dresner SM. Early complications after Ivor Lewis subtotal esophagectomy with two-field lymphadenectomy: risk factors and management. J Am Coll Surg 2002;194:285-97.

9. Tachibana M, Kinugasa $S$, Yoshimura $H$, et al. Clinical outcomes of extended esophagectomy with three-field lymph node dissection for esophageal squamous cell carcinoma. Am J Surg 2005;189:98-109.

10. Nemoto K, Yamada S, Nishio M, et al. Results of radiation therapy for superficial esophageal cancer using the standard radiotherapy method recommended by the Japanese Society of Therapeutic Radiology and Oncology (JASTRO) Study Group. Anticancer Res 2006;26:1507-12.

11. Ariga $T$, Ogawa $K$, Shimoji $H$, et al. Radical radiotherapy for superficial esophageal cancer: impact of clinical $\mathrm{N}$ stage on survival. Anticancer Res 2012;32:3371-6.

12. Murakami $Y$, Nagata $Y$, Nishibuchi I, et al. Long-term outcomes of intraluminal brachytherapy in combination with external beam radiotherapy for superficial esophageal cancer. Int J Clin Oncol 2012;17:263-71.

13. Oh D, Noh JM, Nam H, Lee $H$, Kim TG, Ahn YC. High-dose radiation therapy alone by moderate hypofractionation for patients with thoracic esophageal squamous cell carcinoma. Medicine (Baltimore) 2016;95:e4591.

14. Endo M, Yoshino K, Kawano T, Nagai K, Inoue H. Clinicopathologic analysis of lymph node metastasis in surgically resected superficial cancer of the thoracic esophagus. Dis Esophagus 2000;13:125-9.

15. Shimada $H$, Nabeya $Y$, Matsubara $H$, et al. Prediction of lymph node status in patients with superficial esophageal carcinoma: analysis of 160 surgically resected cancers. Am J Surg 2006;191:250-4.

16. Wang GQ, Jiao GG, Chang FB, et al. Long-term results of operation for 420 patients with early squamous cell esophageal carcinoma discovered by screening. Ann Thorac Surg 2004;77:1740-4.

17. Tachibana M, Hirahara N, Kinugasa S, Yoshimura H. Clinicopathologic features of superficial esophageal cancer: results of consecutive 100 patients. Ann Surg Oncol 2008;15:104-16.

18. Shimizu Y, Tsukagoshi H, Fujita M, Hosokawa M, Kato M, Asaka M. Long-term outcome after endoscopic mucosal resection in patients with esophageal squamous cell carcinoma invading the muscularis mucosae or deeper. Gastrointest Endosc 2002;56:387-90.

19. Akutsu $Y$, Uesato $M$, Shuto $K$, et al. The overall prevalence of metastasis in T1 esophageal squamous cell carcinoma: a retrospective analysis of 295 patients. Ann Surg 2013;257:1032-8.

20. Moraca RJ, Low DE. Outcomes and health-related quality of life after esophagectomy for high-grade dysplasia and intramucosal cancer. Arch Surg 2006;141:545-51.

21. Viklund $P$, Wengstrom $Y$, Rouvelas I, Lindblad $M$, Lagergren J. Quality of life and persisting symptoms after oesophageal cancer surgery. Eur J Cancer 2006;42:1407-14.

22. Koide $Y$, Kodaira T, Tachibana $H$, et al. Clinical outcome of definitive radiation therapy for superficial esophageal cancer. Jpn J Clin Oncol 2017;47:393-400.

23. Cooper JS, Guo MD, Herskovic A, et al. Chemoradiotherapy of locally advanced esophageal cancer: long-term follow-up of a prospective randomized trial (RTOG 85-01). Radiation Therapy Oncology Group. JAMA 1999;281:1623-7.

24. Minsky BD, Pajak TF, Ginsberg RJ, et al. INT 0123 (Radiation Therapy Oncology Group 94-05) phase III trial of combinedmodality therapy for esophageal cancer: high-dose versus standard-dose radiation therapy. J Clin Oncol 2002;20:116774.

25. Ma JB, Song YP, Yu JM, et al. Feasibility of involved-field 
conformal radiotherapy for cervical and upper-thoracic esophageal cancer. Onkologie 2011;34:599-604.

26. Nakagawa S, Kanda T, Kosugi S, Ohashi M, Suzuki T, Hatakeyama K. Recurrence pattern of squamous cell carcinoma of the thoracic esophagus after extended radical esophagectomy with three-field lymphadenectomy. J Am Coll Surg 2004;198:205-11.

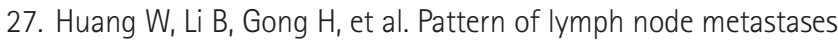
and its implication in radiotherapeutic clinical target volume in patients with thoracic esophageal squamous cell carcinoma: a report of 1077 cases. Radiother Oncol 2010;95:229-33. 